COMMENTARY

\title{
Rituximab in Graves' disease
}

\author{
Patrice Rodien \\ INSERM U694, Centre Hospitalo-Universitaire d'Angers, Endocrinologie, Université d'Angers, 4, rue Larrey, F-49000 Angers, France \\ (Correspondence should be addressed to P Rodien; Email: parodien@chu-angers.fr)
}

Graves' disease, in 2008, is still a mysterious and capricious disease. A tremendous amount of work has been produced and progress achieved in the understanding of its mechanisms. Endocrinologists have very efficient drugs to control hyperthyroidism; they have endeavoured to optimize the regimen of the treatment (dose, duration, titration or combined block-replace therapy), but are still facing 30-60\% relapses in extended follow-up. Moreover, they are still very bad at predicting who will relapse and who will not (1).

Recently, because Graves' disease is, admittedly, a B-cell driven organ specific autoimmune disease, it was proposed to deplete the B-lymphocyte population so as to treat it more rationally and, hopefully, more efficiently $(2,3)$. At first, three patients with Graves' ophthalmopathy were successfully treated with Rituximab, a mouse-human chimeric MAB targeting the CD20 B lymphocytes $(4,5)$. Then an open study confirmed that the drug could bring some benefit to the patients, without any effect on thyroid function (6). A controlled randomized trial in Graves' hyperthyroidism was then conducted, suggesting that rituximab has some potential for reducing the risk of relapses after a short-course treatment with thionamides (7).

Heemstra et al. report the results of a phase II trial of rituximab in relapsing Graves' disease, aiming at controlling hyperthyroidism and reducing the number of further relapses (8). We believe, at the European Journal of Endocrinology, that clinical trials have to be reported, should they end in a therapeutic breakthrough and improvement for patients, in a frustration or, more frequently, in mixed conclusions and new questions.

\section{What does this study tell us?}

(1) Severe relapses of hyperthyroidism in Graves' disease are not controlled by rituximab, at least in the regimen used here.

(2) Nine patients, out of the 13 relapsing patients enrolled in the study, were still euthyroid 14-27 months after the treatment. This is encouraging, considering the chances of cure after a first relapse (1). This positive result, in keeping with those of El Fassi et al. (7), contrasts with the results of Salvi et al. (6).

\section{What cannot be told by this study?}

(1) All of these nine patients had a very mild relapse, some with increasing but normal free tri-iodothyronine/thyroxine. Would these patients have stayed subclinically hyperthyroid, in absence of treatment, or would they have turned to overt hyperthyroidism?

(2) If only mild relapses, and even subclinical relapses, can be treated or prevented by rituximab, how can we identify the patients more likely to benefit from the treatment? No clear relationship between the level of thyrotropin-binding inhibitory immunoglobulin (TBII) and the outcome could be found, but the highest values of TBII were found in the nonresponders. This is reminiscent of the lowest value of TBII in the responders in the El Fassi study (7). The absence of correlation may be due to not only the small numbers of patients but also the poor performance of the TBII in the prediction of relapses after the withdrawal of antithyroid drugs (1).

(3) Similarly, the decrease in CD20 lymphocytes is not predictive of the outcome of the patients, as found also in the Salvi study (6). Circulating lymphocytes do not give a reliable figure of the thyroid housed lymphocytes. The effect of rituximab on B lymphocytes in the thyroid is also disputed $(9,10)$. Again, how then could we accurately identify putative responders?

\section{Where do we stand now?}

When pooling all the patients treated with rituximab for Graves' disease, in the several small sized studies, including this one, we still end up with a limited number of patients, which precludes any definite conclusion. As stated by Heemstra et al. (8), there is a need for a controlled, randomized trial.

By carefully looking at published data, and knowing the fickleness of Graves' disease, it appears that such a trial, if it were to be set up, would have to be controlled for many putative confounding factors such as corticosteroids, radioiodine, duration and regimen of antithyroid drugs, severity and duration of hyperthyroidism, number of relapses and duration of remissions, titre of TBII and rather of bioactivity of TSHR antibodies, smoke, iodine intake; all of them are susceptible to influence the outcome of the patients. 
However, is it worth treating a benign disease like Graves' disease with such a sophisticated and expensive therapeutic? Deciding this for Graves' ophthalmopathy, which can be a devastating condition, is quite easy; hyperthyroidism in Graves' disease deserves a more balanced answer. Relapses of hyperthyroidism can be successfully treated by radioiodine or surgery. It should not be overlooked, however, that Graves' disease goes along with a long-term deterioration of quality of life (11). Most of the time radioiodine and surgery replace hyperthyroidism by hypothyroidism with its own drawbacks. The cost of therapeutics such as rituximab may be a curb to their use in Graves' disease, but relapses of hyperthyroidism and iatrogenic hypothyroidism also represent an economic burden through repeated medical examination, laboratory tests, hospitalization, sickness absences and loss of productivity.

Nevertheless, immunosuppressive therapeutics, even as narrowly targeted as possible, may have immediate or long-term side effects. It is therefore mandatory to unequivocally demonstrate that they bring more benefits than disadvantages, whatever the latter are.

So, let us wait for a controlled, randomized trial.

\section{References}

1 Weetman AP. Graves' disease. New England Journal of Medicine 2000343 1236-1248.

2 Hasselbalch HC. B-cell depletion with Rituximab - a targeted therapy for Graves' disease and autoimmune thyroiditis. Іттиnology Letters $2003 \mathbf{8 8} 85-86$.

3 El Fassi D, Nielsen CH, Hasselbalch HC \& Hegedus L. The rationale for B lymphocyte depletion in Graves' disease. Monoclonal antiCD20 antibody therapy as a novel treatment option. European Journal of Endocrinology 2006154 623-632.
4 Salvi M, Vannucchi G, Campi I, Rossi S, Bonara P, Sbrozzi F, Guastella C, Avignone S, Pirola G, Ratiglia R \& Beck-Peccoz P. Efficacy of rituximab treatment for thyroid-associated ophthalmopathy as a result of intraorbital B-cell depletion in one patient unresponsive to steroid immunosuppression. European Journal of Endocrinology 2006154 511-517.

5 El Fassi D, Nielsen CH, Hasselbalch HC \& Hegedus L. Treatment-resistant severe, active Graves' ophthalmopathy successfully treated with B lymphocyte depletion. Thyroid $200616709-710$.

6 Salvi M, Vannucchi G, Campi I, Curro N, Dazzi D, Simonetta S, Bonara P, Rossi S, Sina C, Guastella C, Ratiglia R \& Beck-Peccoz P. Treatment of Graves' disease and associated ophthalmopathy with the anti-CD20 monoclonal antibody Rituximab: an open study. European Journal of Endocrinology 2007156 33-40.

7 El Fassi D, Nielsen CH, Bonnema SJ, Hasselbalch HC \& Hegedus L. B lymphocyte depletion with the monoclonal antibody Rituximab in Graves' disease: a controlled pilot study. Journal of Clinical Endocrinology and Metabolism 200792 1769-1772.

8 Heemstra K, Toes R, Sepers J, Pereira A, Corssmit E, Huizinga T, Romijn J \& Smit J. Rituximab in relapsing Graves' disease. European Journal of Endocrinology 2008.

9 El Fassi D, Clemmensen O, Nielsen CH, Silkiss RZ \& Hegedus L. Evidence of intrathyroidal B-lymphocyte depletion after Rituximab therapy in a patient with Graves' disease. Journal of Clinical Endocrinology and Metabolism $2007 \mathbf{9 2}$ 3762-3763.

10 Bonara P, Vannucchi G, Campi I, Rossi S, Cantoni F, Frugoni C, Sbrozzi F, Guastella C, Avignone S, Beck-Peccoz P \& Salvi M. Rituximab induces distinct intraorbital and intrathyroidal effects in one patient satisfactorily treated for Graves' ophthalmopathy. Clinical Reviews in Allergy $\mathcal{E}$ Immunology 200834 118-123.

11 Abraham-Nordling M, Torring O, Hamberger B, Lundell G, Tallstedt L, Calissendorff J \& Wallin G. Graves' disease: a longterm quality-of-life follow up of patients randomized to treatment with antithyroid drugs, radioiodine, or surgery. Thyroid 200515 1279-1286.

Received 14 August 2008

Accepted 15 August 2008 